Pacific Journal of Mathematics

A DUAL GEOMETRIC CHARACTERIZATION OF BANACH
SPACES NOT CONTAINING $l_{1}$ 


\title{
A DUAL GEOMETRIC CHARACTERIZATION OF BANACH SPACES NOT CONTAINING $l_{1}$
}

\author{
Elias SaAb and Paulette SaAB
}

\begin{abstract}
It is shown that a Banach space $E$ does not contain a copy of $l_{1}$ if and only if every bounded subset of $E^{*}$ is $w^{*}$-dentable in $\left(E^{*}, \sigma\left(E^{*}, E^{* *}\right)\right)$. The notion of $w^{*}$-scalarly dentable sets in dual Banach space is introduced and it is proved that a Banach space $E$ does not contain a copy of $l_{1}$ if and only if every bounded set in $E^{*}$ is $w^{*}$-scalarly dentable. Finally, a point of continuity criterion that characterizes Asplund operators and those operators that factor through Banach spaces not containing copies of $l_{1}$, is given.
\end{abstract}

Introduction. In [11], [13] Rosenthal and Odell showed that a separable Banach space $E$ does not contain an isomorphic copy of $l_{1}$ if and only if every element $x^{* *} \in E^{* *}$ is Baire-1 when restricted to $\left(B_{E^{*}}, \sigma\left(E^{*}, E\right)\right.$ ). Haydon [6] showed that a Banach space $E$ (separable or not) does not contain a copy of $l_{1}$ if and only if every element $x^{* *} \in E^{* *}$ is universally measurable when restricted to $\left(B_{E^{*}}, \sigma\left(E^{*}, E\right)\right)$.

In this paper we first show that if $E$ is any Banach space such that for every $x^{* *} \in E^{* *}$ and for every $w^{*}$-compact subset $M$ of $B_{E^{*}}$, the restriction of $x^{* *}$ to $\left(M, \sigma\left(E^{*}, E\right)\right)$ has a point of continuity then $E$ contains no copy of $l_{1}$.

In [10] Namioka and Phelps showed that a dual Banach space $E^{*}$ has the Radon-Nikodym property if and only if every bounded subset of $E^{*}$ is $w^{*}$-dentable in $\left(E^{*},\|\|\right)$. Here we shall show that a Banach space $E$ does not contain a copy of $l_{1}$ or equivalently $E^{*}$ has the weak Radon-Nikodym property if and only if every bounded subset of $E^{*}$ is $w^{*}$-dentable in $\left(E^{*}, \sigma\left(E^{*}, E^{* *}\right)\right)$.

To do this we show that a Banach space $E$ does not contain a copy of $l_{1}$ if and only if for every $x^{* *} \in E^{* *}$ and for every $w^{*}$-compact convex subset $C$ of $E^{*}$, the set of points of continuity of $x^{* *}$ restricted to $\left(C, \sigma\left(E^{*}, E\right)\right)$, that are extreme points of $C$ is a $G_{\delta}$ dense subset of $\left(\operatorname{Ext}(C), \sigma\left(E^{*}, E\right)\right)$, where $\operatorname{Ext}(C)$ denotes the set of extreme points of $C$. On the way of proving that we show that a Banach space does not contain a copy of $l_{1}$ if and only if every bounded set in $E^{*}$ is $w^{*}$-scalarly dentable. 
Finally, we give a point of continuity criterion that characterizes Asplund operators and those operators that factor through a Banach space not containing copies of $l_{1}$.

Preliminaries. Let $X$ be a topological Hausdorff space and $f$ be a real valued function on $X$. If $A \subset X$, the oscillation of $f$ on $A$ is defined by $O(f, A)=\sup \{|f(y)-f(x)|, x \in A, y \in A\}$ and the oscillation of $f$ at a point $x$ is given by $O(f, x)=\inf \{O(f, U), U$ open, $x \in U\}$. It is clear that $f$ is continuous at $x$ if and only if $O(f, x)$ is equal to zero. The function is said to be Baire- 1 if $f$ is the pointwise limit of a sequence of continuous functions on $X$. A Banach space $E$ is said to contain a copy of a Banach space $F$ if $F$ is isomorphic to a subspace of $E$, we also say that $F$ embeds into $E$. The closed unit ball of a Banach space $E$ is denoted by $B_{E}$. If $A$ is a subset of the dual $E^{*}$, we denote by $w^{*}-\bar{A}$ the weak* closure of $A$ and by norm $-\bar{A}$ its norm closure, the convex hull of $A$ will be denoted by $\operatorname{conv}(A)$. The symbol $(A, \tau)$ will mean $A$ endowed with the topology $\tau$. If $C$ is a convex set in a Banach space, the set of its extreme points will be denoted by $\operatorname{Ext}(C)$. If $B$ is a bounded subset of a Banach space $E$, an open slice of $B$ is a set of the form

$$
S(A, F, \alpha)=\left\{x \in A ; f(x)>\sup _{A} f-\alpha\right\},
$$

for some $f \neq 0, f \in E^{*}$ and some $\alpha>0$. If $E=F^{*}$ is a dual Banach space and $f \in F$, the slice is called a $w^{*}$-open slice. A closed convex bounded subset $C$ of a Banach space $E$ is said to have the Radon-Nikodym property "RNP" (resp., the weak Radon-Nikodym property "WRNP") if for any bounded linear operator $T: L^{1}[0,1] \rightarrow E$ such that $T\left(1_{A} / \lambda(A)\right) \in C$ for any Lebesgue measurable set $A$ whose Lebesgue measure $\lambda(A) \neq 0$, the operator $T$ is represented by a Bochner kernel (resp., by a Pettis-kernel) $f$ taking its values in $C$. We also call such a set $C$ an RNP set (resp., a WRNP set). If the unit ball of $E$ has the RNP (resp., the WRNP) we say that $E$ has the RNP (resp., the WRNP). For more about RNP and WRNP we refer the reader to [4], [8], [5] and [12].

The Banach spaces $l_{1}, c_{0}, l_{\infty}$ will have their usual meaning. A sequence $\left(x_{n}\right)_{n \geq 1}$ in a Banach space is said to be equivalent to the usual $l_{1}$-basis if there is a $\delta>0$, such that

$$
\left\|\sum_{n=1}^{k} a_{n} x_{n}\right\| \geq \sum_{n=1}^{k}\left|a_{n}\right|,
$$


for any $k \geq 1$ and any scalars $a_{1}, a_{2}, \ldots, a_{k}$. All Banach spaces considered are over the real field.

If $T: E \rightarrow F$ is a bounded linear operator, $T^{*}: F^{*} \rightarrow E^{*}$ will always denote the adjoint of $T$.

Let $E$ be a Banach space, let $E^{*}$ be its dual and $E^{* *}$ its bidual. Let us consider the following two properties of $E$.

(P1)-For every $x^{* *}$ in $E^{* *}$, the restriction of $x^{* *}$ to $\left(B_{E^{*}}, \sigma\left(E^{*}, E\right)\right)$ is a Baire-1 function on $\left(B_{E^{*}}, \sigma\left(E^{*}, E\right)\right)$.

(P2)-For every $x^{* *}$ in $E^{* *}$ and for every closed subset $M$ of $\left(B_{E^{*}}, \sigma\left(E^{*}, E\right)\right)$, the restriction of $x^{* *}$ to $M$ has a point of continuity.

It is easy to see that (P1) implies (P2), the converse is not true in general, an example will be provided later. In [11] Odell and Rosenthal showed that if $E$ is any Banach space that does not contain a copy of $l_{1}$ then $E$ satisfies (P2) and if $E$ is in addition separable then $\left(B_{E^{*}}, \sigma\left(E^{*}, E\right)\right)$ is metrizable and therefore (P2) implies (P1) by the Baire charaterization theorem [1].

In what follows, we will show that if $E$ is any Banach space that satisfies (P2), then $E$ does not contain any copy of $l_{1}$. First,' we need the following two propositions.

Proposition 1. Let $X$ be a compact Hausdorff space and $f$ be a real valued function on $X$ such that for every $\varepsilon>0$, and every closed subset $A$ of $X$, there exists an open subset $U$ of $X$ such that $U \cap A \neq \varnothing$ and $O(f, U \cap A) \leq \varepsilon$. Then the set of points of continuity of $f$ is a $G_{\delta}$ dense subset of $X$.

Proof. Let $n \geq 1$ be an integer and consider the set $Z_{n}=\{x \in X, x$ has an open neighborhood $U$ such that $O(f, U) \leq 1 / n\}$. It is clear that $Z_{n}$ is open. We will show that $Z_{n}$ is dense in $X$. To this end, let $W$ be a non-empty open subset of $X$ and let $A=\bar{W}$ be the closure of 
$W$ in $X$. Choose an open set $U$ such that $U \cap A \neq \varnothing$ and $O(f, U \cap A) \leq$ $1 / n$. It is clear that $U \cap W \neq \varnothing$ and any $v \in U \cap W$ belongs to $Z_{n}$. Hence $W \cap Z_{n} \neq \varnothing$. Therefore $Z_{n}$ is dense in $X$. Apply the Baire Category Theorem to conclude that the set

$$
Z=\bigcap_{n=1}^{\infty} Z_{n}
$$

is a $G_{\delta}$ dense subset of $X$. The set $Z$ is precisely the set of points of continuity of $f$.

Proposition 2. Let $E$ be a Banach space that satisfies (P2) and $H$ be any Banach space. If $L: H \rightarrow E$ is a bounded linear operator, then for every $w^{*}$-compact subset $M$ of $E^{*}$ and every $x^{* *} \in H^{* *}$, the restriction of $x^{* *}$ to $\left(L^{*}(M), \sigma\left(H^{*}, H\right)\right)$ has a point of continuity.

Proof. Let $L^{*}: E^{*} \rightarrow H^{*}$ and let $M$ be a $w^{*}$-compact subset in $E^{*}$ and $x^{* *} \in H^{* *}$. Let $B$ be a $w^{*}$-compact subset of $L^{*}(M)$. By Proposition 1 it is enough to show that $B$ contains a $w^{*}$-relatively open non-empty subset on which $x^{* *}$ has arbitrarily small oscillation. To this end, let $\varepsilon>0$ and let $A=L^{*^{-1}}(B) \cap M$, then $A$ is a $w^{*}$-compact subset of $E^{*}$ satisfying $L^{*}(A)=B$. Let $A_{1}$ be a minimal (under inclusion) $w^{*}$-compact subset of $E^{*}$ such that $L^{*}\left(A_{1}\right)=B$. The linear functional $x^{* *} L^{*}$ belongs to $E^{* *}$, therefore by hypothesis $A_{1}$ contains a $w^{*}$-relatively open set $W$ such that $O\left(x^{* *} L^{*}, W\right) \leq \varepsilon$. Let $B_{1}=L^{*}\left(A_{1} \backslash W\right)$, the set $B_{1}$ is a $w^{*}$-compact subset of $H^{*}$ and $B_{1} \neq B$ by the minimality of $A_{1}$. Let $u$ and $v$ be elements in $B \backslash B_{1}$, there exists $u_{1}$ and $v_{1}$ in $W$ such that $u=L^{*}\left(u_{1}\right)$ and $v=L^{*}\left(v_{1}\right)$. Notice that

$$
\left|x^{* *}(u)-x^{* *}(v)\right|=\left|x^{* *} L^{*}\left(u_{1}\right)-x^{* *} L^{*}\left(v_{1}\right)\right| \leq O\left(x^{* *} L^{*}, W\right) \leq \varepsilon .
$$

This shows that $O\left(x^{* *}, B \backslash B_{1}\right) \leq \varepsilon$, and finishes the proof of the proposition. theorem.

With the help of the above proposition we obtain the following

THEOREM 3. Let E be a Banach space. The following statements are equivalent:

(i) The space $E$ does not contain any copy of $l_{1}$;

(ii) For every $x^{* *}$ in $E^{* *}$ and for every $w^{*}$-compact subset $M$ of $E^{*}$, the restriction of $x^{* *}$ to $M$ has a point of continuity when $M$ is endowed with the relative $w^{*}$-topology $\sigma\left(E^{*}, E\right)$. 
Proof. All we have to show is (ii) $\rightarrow$ (i). Suppose that (ii) holds and $E$ contains a copy of $l_{1}$. Let $H: l_{1} \rightarrow E$ be the isomorphic embedding of $l_{1}$ into $E$. Then $L^{*}: E^{*} \rightarrow l_{\infty}$ is onto. Let $C=B_{l_{\infty}}$ denote the unit ball of $l_{\infty}$. Let $h \in l_{\infty}^{*}$ and $M$ be a $w^{*}$-compact subset of $C$. Proposition 2 implies that the restriction of $h$ to $\left(M, \sigma\left(l_{\infty}, l_{1}\right)\right)$ has a point of continuity. By the Baire Charaterization Theorem [1] ( $C$ is metrizable) $h$ will be a Baire-1 function on $C$. But this shows that any $h \in l_{\infty}^{*}$ is Baire-1 on $C$, and this is a contradiction since any $h \in l_{\infty}^{*} \backslash l_{1}$ is not Baire-1 on $C$.

Example of a Banach space $E$ that satisfies (P2) but not (P1). [11].

Let $E=c_{0}(\Gamma)$ where $\Gamma$ is uncountable. Because $l_{1}$ does not embed into $E, E$ satisfies (P2). Let $K$ be the unit ball of $l_{1}(\Gamma)$, and let $x^{* *}=$ $\left(u_{\alpha}\right)_{\alpha \in \Gamma} \in l_{\infty}(\Gamma)$ if $x^{* *}$ restricted to $K$ is Baire-1, then $x^{* *}$ will be the $w^{*}$-limit of a sequence in $E=c_{0}(\Gamma)$, therefore $x^{* *}$ will be countably supported. This shows that $E$ does not satisfy (P1).

Definition 4. Let $A$ be a bounded subset of $E^{*}$. We say that $A$ is $w^{*}$-scarlarly dentable if for every $\varepsilon>0$ and every $x^{* *} \in E^{* *}$ there exists a $w^{*}$-open slice $S$ of $A$ such that $O\left(x^{* *}, S\right) \leq \varepsilon$.

The above definition should be compared to the following definition of $w^{*}$-dentability [10].

Definition 5. Let $A$ be a bounded subset of $E^{*}$. We say that $A$ is $w^{*}$-dentable, if for every $\varepsilon>0$, there exists a $w^{*}$-open slice $S$ of $A$ such that the norm diameter of $S$ is less than $\varepsilon$.

TheORem 6. Let $E$ be a Banach space and let $E^{*}$ be its dual. The following statements are equivalent:

(i) The space $E$ does not contain a copy of $l_{1}$;

(ii) Every non-empty bounded subset of $E^{*}$ is $w^{*}$-scalarly dentable;

(iii) Every non-empty $w^{*}$-compact convex subset of $E^{*}$ is $w^{*}$-scalarly dentable;

(iv) For every non-empty bounded subset $A$ of $E^{*}$ and every $x^{* *}$ in $E^{* *}, A$ contains a non-empty $w^{*}$-relatively open subset of $E^{*}$ on which $x^{* *}$ has arbitrarily small oscillation;

(v) For every non-empty $w^{*}$-compact subset $A$ of $E^{*}$ and every $x^{* *}$ in $E^{* *}, A$ contains a non-empty $w^{*}$-relatively open subset of $E^{*}$ on which $x^{* *}$ has arbitrarily small oscillation;

(vi) For every non-empty $w^{*}$-compact subset $A$ of $E^{*}$ and every $x^{* *}$ in $E^{* *}$ the restriction of $x^{* *}$ to $\left(A, \sigma\left(E^{*}, E\right)\right)$ has a point of continuity. 
(vii) For every non-empty $w^{*}$-compact subset $A$ of $E^{*}$ and every $x^{* *}$ in $E^{* *}$ the set of points of continuity of $x^{* *}$ restricted to $\left(A, \sigma\left(E^{*}, E\right)\right)$ is a $w^{*}$-dense $G_{\delta}$ subset of $\left(A, \sigma\left(E^{*}, E\right)\right)$.

Proof. (i) $\leftrightarrow$ (vi) is Theorem 3 .

(ii) $\rightarrow$ (iii), (iv) $\rightarrow$ (v) and (vii) $\rightarrow$ (vi) are evident.

(iii) $\rightarrow$ (iv) by taking $C=w^{*}-\overline{\operatorname{conv}}(A)$.

(v) $\rightarrow$ (vii) is Proposition 1. All that remains is to prove that (vi) $\rightarrow$ (ii).

Let $C=w^{*}-\overline{\operatorname{conv}}(A)$, let $x^{* *} \in E^{* *}$ and let $f$ be the restriction of $x^{* *}$ to $C$. Consider the set

$$
Z=\{u \in C ; O(f, u) \geq \varepsilon\} .
$$

It is easy to see that $Z$ is a $w^{*}$-closed subset of $C$. The set $Z$ is also convex, for let $u$ and $v$ be two elements in $Z$ and let $0 \leq \alpha \leq 1$. Consider $W$ a $w^{*}$-open subset such that $\alpha u+(1-\alpha) v \in W \cap C$. Choose $U$ and $V$ two $w^{*}$-open neighborhoods of $u$ and $v$ respectively such that $(\alpha U+(1-\alpha) V) \cap C \subset W \cap C$. It follows that $O(f, U \cap C) \geq \varepsilon$ and $O(f, V \cap C) \geq \varepsilon$. Therefore $O(f, W \cap C) \geq \varepsilon$. Hence $\alpha u+(1-\alpha) v$ $\in Z$. If $\operatorname{Ext}(C) \subset Z$, then by the Krein-Milman theorem $Z=C$ but $Z \neq C$ because $f$ has a point of continuity. Let $e \in \operatorname{Ext}(C)$ such that $e$ does not belong to $Z$. This means $O(f, e)<\varepsilon$. Let $U$ be a $w^{*}$-closed convex neighborhood of $e$ such that $O(f, U \cap C) \leq \varepsilon$. By the extremality of $e$, there exists a $w^{*}$-open slice $S$ of $C$ ([2], Theorem 25.13) such that $e \in S \subset U \cap C$. It is easy to see that $S \cap A \neq \varnothing$. Hence $O(f, S \cap A) \leq$ $\varepsilon$. In fact we have more, since

$$
w^{*}-\overline{\operatorname{conv}}(S \cap A) \subset U \cap C,
$$

then

$$
O\left(f, w^{*}-\overline{\operatorname{conv}}(S \cap A)\right) \leq \varepsilon .
$$

This completes the proof.

In the proof of (vi) $\rightarrow$ (ii) we showed the following fact that we state as a proposition.

Proposition 7. Let $A$ be a bounded subset of $E^{*}, x^{* *}$ be an element of $E^{* *}$, and let $C=w^{*}-\overline{\operatorname{conv}}(A)$. If $x^{* *}$ restricted to $\left(C, \sigma\left(E^{*}, E\right)\right)$ has a point of continuity, then for any $\varepsilon>0$, there exists a $w^{*}$-open slice $S$ of $C$ such that $A \cap S \neq \varnothing$, and

$$
O\left(x^{* *}, w^{*}-\overline{\operatorname{conv}}(A \cap S)\right) \leq \varepsilon .
$$


REMARK. Using Proposition 7 and a result of Haydon [6] we are going to give another proof of (ii) $\rightarrow$ (i) in Theorem 3 . The argument goes as follows: If $l_{1}$ embeds in $E$, then there exists a $w^{*}$-compact convex subset $C$ in $E^{*}$ such that $C \neq$ norm- $\overline{\operatorname{conv}}(\operatorname{Ext} C)$ [6]. From this fact, Haydon was able to find $x^{* *} \in E^{* *}, \varepsilon>0$ and a bounded non-empty subset $A$ of $E^{*}$ satisfying $O\left(x^{* *}, w^{*}\right.$-conv $\left.(U \cap A)\right) \geq \varepsilon$ for any $w^{*}$-open subset $U$ of $E^{*}$ such that $U \cap A \neq \varnothing$. Apply Proposition 7 to find a contradiction.

Let $E$ be a Banach space not containing $l_{1}$. Let $C$ be a $w^{*}$-compact convex subset of $E^{*}$ and let $x^{* *} \in E^{* *}$. By Theorem 6 we know that the set $Z$ of the points of continuity of $x^{* *}$ restricted to $\left(C, \sigma\left(E^{*}, E\right)\right)$ is a $G_{\delta}$ dense subset of $\left(C,\left(E^{*}, E\right)\right)$. A question can be asked: Does $Z$ contain any extreme point of $C$ ? In the next proposition we will give an affirmative answer to this question. In fact we have more.

The proof of the next proposition uses the idea of ([9] Theorem 2.2) and Proposition 7.

Proposition 8. With the above notations, the set $Z \cap \operatorname{Ext}(C)$ is $a G_{\delta}$ dense subset of $\left(\operatorname{Ext}(C), \sigma\left(E^{*}, E\right)\right)$ and consequently $C=w^{*}$ $\overline{\operatorname{conv}}(Z \cap \operatorname{Ext}(C))$.

Proof. Let $X=\operatorname{Ext}(C)$ and $\varepsilon>0$. Let $B_{\varepsilon}=\left\{u \in X\right.$; $u$ has a $w^{*}$-open neighborhood $V$ such that $\left.O\left(x^{* *}, C \cap V\right) \leq \varepsilon\right\}$. The set $B_{\varepsilon}$ is open in $\left(X, \sigma\left(E^{*}, E\right)\right)$. It is also dense in $\left(X, \sigma\left(E^{*}, E\right)\right)$. For, let $W$ be a $w^{*}$-open subset such that $W \cap X \neq \varnothing$. Let $D=w^{*}-\bar{X}$ and let $A=W \cap D$. By Proposition 7, there exists a $w^{*}$-open subset $U$ such that $U \cap A \neq \varnothing$ and $O\left(x^{* *}, w^{*}-\overline{\operatorname{conv}}(U \cap A)\right) \leq \varepsilon / 2$. Let $V=U \cap W$, then $\varnothing \neq V \cap D \subset$ $W \cap D$ and $O\left(x^{* *}, w^{*}-\overline{\operatorname{conv}}(V \cap D)\right) \leq \varepsilon / 2$. From now on the proof goes as in ([9], Theorem 2.2) with some obvious changes.

Corollary 9. A Banach space $E$ does not contain a copy of $l_{1}$ if and only if for every $x^{* *} \in E^{* *}$ and every $w^{*}$-compact convex subset $C$ in $E^{*}$, the intersection $Z \cap \operatorname{Ext}(C)$ of the set $Z$ of the points of continuity of $x^{* *}$ restricted to $\left(C, \sigma\left(E^{*}, E\right)\right)$ with the extreme points of $C$ is a dense $G_{\delta}$ subset of $\left(\operatorname{Ext}(C), \sigma\left(E^{*}, E\right)\right)$ and $C=w^{*}-\overline{\operatorname{conv}}(Z \cap \operatorname{Ext}(C))$.

If $(X, \tau)$ is a locally convex Hausdorff topological vector space, and $A$ is a bounded subset of $(X, \tau)$, the set $A$ is said to be dentable if for every zero-neighborhood $V$ in $X$, there exists an open slice $S$ of $A$ such that 
$S-S \subset V$. We say that $(X, \tau)$ is dentable if every bounded subset of $(X, \tau)$ is dentable. It is clear from this definition that a subspace (closed or not) of a dentable space is dentable.

If $A$ is a bounded subset of $E^{*}$, the dual of a Banach space $E$, let us agree to say that $A$ is $w^{*}$-dentable in $\left(E^{*}, \sigma\left(E^{*}, E^{* *}\right)\right)$, if for any $\sigma\left(E^{*}, E^{* *}\right)$ zero-neighborhood $V$ in $E^{*}$, there exists a $w^{*}$-open slice $S$ such that $S-S \subset V$, accordingly, the set $A$ is $w^{*}$-dentable in $\left(E^{*},\|\|\right)$ if $A$ is $w^{*}$-dentable in the sense of Definition 5.

In [10] Namioka and Phelps showed that the dual $E^{*}$ of a Banach space $E$ has the RNP if and only if every non-empty bounded subset of $E^{*}$ is $w^{*}$-dentable in $\left(E^{*},\|\|\right)$. It turns out, as we shall soon show, that $E^{*}$ has the WRNP if and only if every non-empty bounded subset of $E^{*}$ is $w^{*}$-dentable in $\left(E^{*}, \sigma\left(E^{*}, E^{* *}\right)\right)$.

THEOREM 10. For a Banach space $E$, the following statements are equivalent:

(i) The space $E$ does not contain a copy of $l_{1}$;

(ii) Every non-empty bounded subset $A$ in $E^{*}$ is $w^{*}$-scalarly dentable;

(iii) Every non-empty bounded subset $A$ in $E^{*}$ is $w^{*}$-dentable in $\left(E^{*}, \sigma\left(E^{*}, E^{* *}\right)\right)$.

Proof. All we have to show is (i) implies (iii). For this, let $A$ be a bounded subset of $E^{*}$ and $V$ be a $\sigma\left(E^{*}, E^{* *}\right)$ zero-neighborhood in $E^{*}$, the set $V$ has the form

$$
V=\left\{x^{*} \in E^{*} ;\left|x_{i}^{* *}\left(x^{*}\right)\right| \leq \varepsilon, x_{i}^{* *} \in E^{* *}, i=1,2, \ldots, n\right\} .
$$

Let $C=w^{*}-\overline{\operatorname{conv}}(A)$, and let $Z_{i}$ be the set of points of continuity of the restriction $x_{i}^{* *}$ to $\left(C, \sigma\left(E^{*}, E\right)\right), i=1,2, \ldots, n$, and let $T_{i}=Z_{i} \cap \operatorname{Ext}(C)$. By Proposition $8, T_{i}$ is a $G_{\delta}$ dense subset of $\left(\operatorname{Ext}(C), \sigma\left(E^{*}, E\right)\right)$. Hence $T=\bigcap_{i=1}^{n} T_{i}$ is also a $G_{\delta}$ dense subset of $\left(\operatorname{Ext}(C), \sigma\left(E^{*}, E\right)\right)$ since this later is a Baire space by a theorem of Choquet [2]. Let $e \in T$ and choose $U$ a $w^{*}$-neighborhood of $e$ such that $O\left(x_{i}^{* *}, U \cap C\right) \leq \varepsilon$ for $i=1,2, \ldots, n$. By the extremality of $e$ in $C$, choose a $w^{*}$-open slice $S$ of $C$ such that $e \in S \subset U \cap C$. This means that $O\left(x_{i}^{* *}, S\right) \leq \varepsilon$ for $i=1,2, \ldots, n$. Hence $S-S \subset V$. Therefore $S \cap A \neq \varnothing$ is a $w^{*}$-open slice of $A$ and $S \cap A-$ $S \cap A \subset V$. This completes the proof.

It is known that the dual $E^{*}$ of a Banach space $E$ has the WRNP if and only if $E$ does not contain a copy of $l_{1}$ [7]. Combining this fact with Theorem 10 we get 
THEOREM 11. The following statements about a Banach space $E$ are equivalent:

(i) The space $E^{*}$ has WRNP;

(ii) Every non-empty bounded subset of $E^{*}$ is $w^{*}$-scalarly dentable;

(iii) Every non-empty bounded subset of $E^{*}$ is $w^{*}$-dentable in $\left(E^{*}, \sigma\left(E^{*}, E^{* *}\right)\right)$.

REMARK. It is easy to see that for every locally convex Hausdorff space $F$, the space $\left(F, \sigma\left(F, F^{*}\right)\right)$ is dentable, for $\left(F, \sigma\left(F, F^{*}\right)\right)$ can be identified with a subspace of $\mathbf{R}^{F^{*}}$ by the map $h(x)=\left(x^{*}(x)\right)_{x^{*} \in F^{*}}$. The space $\mathbf{R}^{F^{*}}$ is of course dentable. Hence $\left(F, \sigma\left(F, F^{*}\right)\right)$ is also dentable, therefore one cannot replace the statement " $w^{*}$-dentable in $\left(E^{*}, \sigma\left(E^{*}, E^{* *}\right)\right)$ " in (iii) of Theorem 11 by the statement "dentable in $\left(E^{*}, \sigma\left(E^{*}, E^{* *}\right)\right)$ ". This also shows that there is no connection whatsoever between the WRNP for a Banach space $F$ and the dentability of $\left(F, \sigma\left(F, F^{*}\right)\right)$, while the RNP for a Banach space $F$ is equivalent to the dentability of $(F,\|\|)$ see $([4]$, p. 136).

In the following theorems we give a point of continuity criterion that characterizes Asplund operators and those operators that factor through a Banach space not containing $l_{1}$.

THEOREM 12. Let $H$ and $F$ be two Banach spaces, and let $T: H \rightarrow F$ be a bounded linear operator, then the following statements are equivalent:

(i) The operator $T$ factors through a Banach space not containing $l_{1}$;

(ii) For every $w^{*}$-compact convex subset $M$ in $F^{*}$ and every $x^{* *} \in H^{* *}$, the restriction of $x^{* *}$ to $\left(T^{*}(M), \sigma\left(H^{*}, H\right)\right)$ has a point of continuity.

Proof. To see that (i) implies (ii), let $E$ be a Banach space not containing $l_{1}$ and such that $T$ factors through $E$ as follows

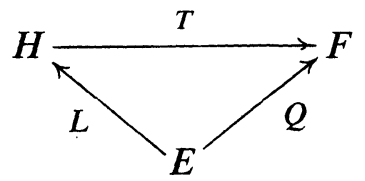

If $M$ is a $w^{*}$-compact subset of $F^{*}$, then $T^{*}(M)=L^{*}\left(Q^{*}(M)\right)$. An appeal to Proposition 2 and Theorem 3 finishes the proof of this implication.

Conversely, it is enough to show that $T\left(B_{H}\right)$ contains no copy of the $l_{1}$-basis and apply the construction of Davis, Figiel, Johnson and Pelczynski [3]. Suppose not and let $\left(x_{n}\right)_{n \geq 1}$ be a sequence in $T\left(B_{H}\right)$ equivalent to 
the $l_{1}$-basis. For every $n \geq 1$, choose $y_{n} \in H$ such that $T\left(y_{n}\right)=x_{n}$. It is easy to see that $\left(y_{n}\right)_{n \geq 1}$ is also equivalent to the $l_{1}$-basis. Let $S: l_{1} \rightarrow H$ defined by $S\left(e_{n}\right)=y_{n}$ where $\left(e_{n}\right)_{n \geq 1}$ is the usual basis of $l_{1}$. The map $S^{*} \circ T^{*}: F^{*} \stackrel{T}{\rightarrow} H^{*} \rightarrow l_{\infty}$ is onto. To see this, let $z \in l_{\infty}$ and let $R$ the closed linear span of $\left(x_{n}\right)_{n \geq 1}$. Define $\tilde{u} \in R^{*}$ by $\tilde{u}\left(x_{n}\right)=\left\langle e_{n}, z\right\rangle$. Let $u \in F^{*}$ be an extension of $\tilde{u}$. It is clear that $S^{*} \circ T^{*}(u)=z$. Hence every $w^{*}$-compact subset $N$ of $l_{\infty}$ can be written $N=S^{*}\left(T^{*}(M)\right)$, where $M$ is a $w^{*}$-compact subset of $F^{*}$. Now use (ii) and Proposition 2 to find a contradiction.

Definition 13. A Banach space $G$ is called an Asplund space if $G^{*}$ has the Radon-Nikodym property.

Theorem 12 has to be compared with the following:

THEOREM 14. Let $H$ and $F$ be two Banach spaces and let $T: H \rightarrow F$ be $a$ bounded linear operator, then the following statements are equivalent:

(i) The operator $T$ factors through an Asplund Banach space;

(ii) For every $w^{*}$-compact convex subset $M$ of $F^{*}$ the identity map

$$
\left(T^{*}(M), \sigma\left(H^{*}, H\right)\right) \rightarrow\left(T^{*}(M),\|\|\right)
$$

has a point of continuity.

Proof. Consider the same diagram as in Theorem 12, and suppose that $E$ is an Asplund space, then $T^{*}(M)=L^{*}\left(Q^{*}(M)\right)$ and $Q^{*}(M)$ is an RNP set. Therefore $L^{*}\left(Q^{*}(M)\right)$ is an RNP set [14]. Any $w^{*}$-strongly exposed point [10] of $T^{*}(M)$ is a point of continuity of $\left(T^{*}(M), \sigma\left(H^{*}, H\right)\right) \rightarrow\left(T^{*}(M),\|\|\right)$. Conversely (ii) implies that any $w^{*}$ compact convex subset $C$ of $T^{*}\left(B_{F^{*}}\right)$ contains $w^{*}$-relatively open subsets of arbitrarily small diameter and therefore by [10] $T^{*}\left(B_{F}\right)$ is an RNP set. Apply [15] to finish the proof.

An operator that satisfies one of the above equivalent conditions is called an Asplund operator.

\section{REFERENCES}

1. R. Baire, Sur les fonctions des variables, réelles, Ann. Math. Pure Appl., Serie 3, t.3, (1899), 16-30.

2. G. Choquet, Lecture on analysis, Vol. II, Benjamin, New York, (1969).

3. W. J. David, T. Figiel, W. B. Johnson and A. Pelczynski, Factoring weakly compact operators, J. Funct. Anal., 17 (1974), 311-327. 
4. J. Diestel and J. J. Uhl, Vector measures, Math. Surveys 15, American Math. Soc., Providence, (1977).

5. N. Ghoussoub and E. Saab, On the weak Radon-Nikodym property, Proc. Amer. Math. Soc., 81 (1981), 81-84.

6. R. Haydon, Some more characterizations of Banach spaces containing $l_{1}$, Math Proc. Cambridge Philos. Soc., 80 (1976), 269-276.

7. L. Janicka, Wlasnosci Typu Radona-Nikodyma dla Przestrzeni Banacha, Thesis, 1978, Wroclaw.

8. K. Musial, The weak Radon-Nikodym property for Banach spaces, Studia Math., 64 (1978), 151-174.

9. I. Namioka, Neighborhoods of extreme points, Israel J. Math., 5 (1967), 145-152.

10. I. Namioka and R. R. Phelps, Banach spaces which are Asplund spaces, Duke Math. J., 42 (1975),735-750.

11. E. Odell and H. P. Rosenthal, A double dual characterization of separable Banach spaces containing $l_{1}$, Israel J. Math., 20 (1975), 375-384.

12. L. H. Riddle, E. Saab and J. J. Uhl, Jr., Sets with weak Radon-Nikodym property in dual Banach spaces, to appear in Indiana Univ. Math. J.

13. H. P. Rosenthal, Pointwise compact subsets of first Baire class, Amer. J. Math., 99 (1977), 362-378.

14. E. Saab, A characterization of $w^{*}$-compact convex sets having the Radon-Nikodym property, Bull. Sci. Math., (2) 104 (1980), 79-88.

15. C. Stegall, The Radon-Nikodym property in conjugate Banach spaces II, Trans. Amer. Math. Soc., 264 (1981), 507-519.

Received September 23, 1981.

UNIVERSITY OF MISSOURI-COLUMBIA

Columbia, MO 65211 



\title{
PACIFIC JOURNAL OF MATHEMATICS EDITORS
}

\author{
Donald BabBitT (Managing Editor) \\ University of California \\ Los Angeles, CA 90024 \\ Hugo Rossi \\ University of Utah \\ Salt Lake City, UT 84112 \\ C. C. Moore and Arthur Ogus \\ University of California \\ Berkeley, CA 94720
}

J. DugundiI

Department of Mathematics

University of Southern California

Los Angeles, CA 90089-1113

R. FINN and H. SAMELSON

Stanford University

Stanford, CA 94305

\section{ASSOCIATE EDITORS}
R. ARENS
E. F. BECKENBACH
B. H. NeUMANN
F. WOLF
K. YosHIDA

(1906-1982)

\section{SUPPORTING INSTITUTIONS}

UNIVERSITY OF ARIZONA

UNIVERSITY OF BRITISH COLUMBIA

CALIFORNIA INSTITUTE OF TECHNOLOGY

UNIVERSITY OF CALIFORNIA

MONTANA STATE UNIVERSITY

UNIVERSITY OF NEVADA, RENO

NEW MEXICO STATE UNIVERSITY

OREGON STATE UNIVERSITY

\author{
UNIVERSITY OF OREGON \\ UNIVERSITY OF SOUTHERN CALIFORNIA \\ STANFORD UNIVERSITY \\ UNIVERSITY OF HAWAII \\ UNIVERSITY OF TOKYO \\ UNIVERSITY OF UTAH \\ WASHINGTON STATE UNIVERSITY \\ UNIVERSITY OF WASHINGTON
}

The Supporting Institutions listed above contribute to the cost of publication of this Journal, but they are not owners or publishers and have no responsibility for its content or policies.

Mathematical papers intended for publication in the Pacific Journal of Mathematics should be in typed form or offset-reproduced (not dittoed), double spaced with large margins. Please do not use built up fractions in the text of the manuscript. However, you may use them in the displayed equations. Underline Greek letters in red, German in green, and script in blue. The first paragraph must be capable of being used separately as a synopsis of the entire paper. In particular it should contain no bibliographic references. Please propose a heading for the odd numbered pages of less than 35 characters. Manuscripts, in triplicate, may be sent to any one of the editors. Please classify according to the scheme of Math. Reviews, Index to Vol. 39. Supply name and address of author to whom proofs should be sent. All other communications should be addressed to the managing editor, or Elaine Barth, University of California, Los Angeles, California 90024.

There are page-charges associated with articles appearing in the Pacific Journal of Mathematics. These charges are expected to be paid by the author's University, Government Agency or Company. If the author or authors do not have access to such Institutional support these charges are waived. Single authors will receive 50 free reprints; joint authors will receive a total of 100 free reprints. Additional copies may be obtained at cost in multiples of 50 .

The Pacific Journal of Mathematıcs is issued monthly as of January 1966. Regular subscription rate: $\$ 132.00$ a year (6 Vol., 12 issues). Special rate: $\$ 66.00$ a year to individual members of supporting institutions.

Subscriptions, orders for numbers issued in the last three calendar years, and changes of address should be sent to Pacific Journal of Mathematics, P.O. Box 969, Carmel Valley, CA 93924, U.S.A. Old back numbers obtainable from Kraus Periodicals Co., Route 100, Millwood, NY 10546.

The Pacific Journal of Mathematics ISSN 0030-8730 is published monthly by the Pacific Journal of Mathematics at P.O. Box 969, Carmel Valley, CA 93924. Application to mail at Second-class postage rates is pend ing at Carmel Valley, California, and additional mailing offices. Postmaster: Send address changes to Pacific Journal of Mathematics, P. O. Box 969, Carmel Valley, CA 93924.

PUBLISHED BY PACIFIC JOURNAL OF MATHEMATICS, A NON-PROFIT CORPORATION

Copyright $(1) 1983$ by Pacific Journal of Mathematics 


\section{Pacific Journal of Mathematics}

\section{Vol. 105, No. $2 \quad$ October, 1983}

Spiros Argyros, On compact spaces without strictly positive measure . . . . . 257

Steven Robert Bell, Regularity of the Bergman projection in certain nonpseudoconvex domains ............................273

Carlos R. Borges and Gary Fred Gruenhage, Sup-characterization of

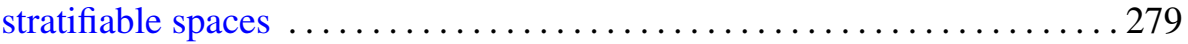

Giuseppe Ceresa and Alberto Collino, Some remarks on algebraic

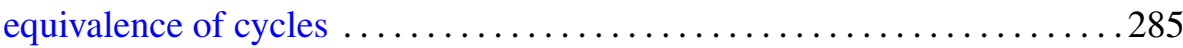

Charles Kam-Tai Chui and Maurice Hasson, Degree of uniform approximation on disjoint intervals ....................... 291

Gary Gundersen, Meromorphic functions that share two finite values with

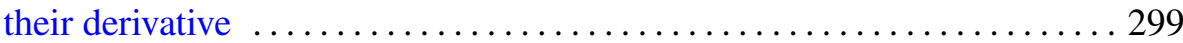

Richard I. Hartley, Lifting group homomorphisms ............... 311

Gerald William Johnson and David Lee Skoug, Notes on the Feynman integral. III. The Schroedinger equation ..................... 321

John Cronan Kieffer, Some topologies on the set of discrete stationary

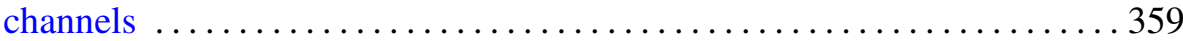

Harald Luschgy and Wolfgang Thomsen, Extreme points in the Hahn-Banach-Kantorovič setting . ............................... 387

Zbigniew Piotrowski, A. Rosłanowski and Brian M. Scott, The pinched-cube topology ................................ 399

Elias Saab and Paulette Saab, A dual geometric characterization of Banach

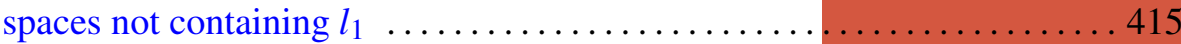

Walter Schachermayer, Norm attaining operators on some classical Banach

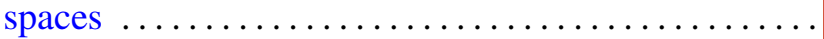

Martin Scharlemann, Essential tori in 4-manifold boundaries

Jacques C. H. Simon, Nonlinear representations of Poincaré group and global solutions of relativistic wave equations ..........

Adrian R. Wadsworth, $p$-Henselian field: $K$-theory, Galois cohomology, and graded Witt rings $\ldots \ldots \ldots \ldots \ldots \ldots \ldots \ldots \ldots$ 